\title{
Bemerkungen zur Stele des Merer in Krakau
}

Discussion of some of the textual problems of the stele of Merer in the Cracow Museum, first published by Cerný in $\mathscr{F E A ~} 47$ ( $196 \mathrm{r}$ ). This important document for the history of the First Intermediate Period contains the earliest attestation of the image of the balance in the Judgement of the Dead.

Die Stele des Mrr in Krakau ${ }^{3}$ ist von J. Černý in Band 47 ( I 96I) dieser Zeitschrift (pp. 5-9; pl. i) veröffentlicht worden. Seitdem ist die Inschrift mehrfach behandelt und kommentiert worden. ${ }^{4}$ Trotzdem sind einige Passagen in ihr dunkel geblieben. Der folgende Artikel versucht sie etwas zu erhellen.

I. Große Schwierigkeiten bereitet haben die Sätze in der ersten Hälfte von Z.4. Die bisherigen Übersetzungen:

Černý: 'I did not spit in the eyes of a good man, of one who spoke or of one who knew, of one who ... or of one who bent (his) arm to me.'

Schenkel: 6 'Ich habe nicht in die Augen gespieen, dem, der mit mir sprach, zu Gefallen, da ich mir des Verletzenden bewußt war und (lieber) den Arm beugte.'

Groll:? 'I was not spat upon in my eyes because of the quality of my speaking and because of my knowledge of one who bent (his) arm to me.'

Lichtheim: ' I was not spat in the eyes, owing to the worth of my speech, the competence of my counsel, and the bending of my arm.'

1 CG 42077: Aldred, New Kingdom Art in Ancient Egypt, no. 49.

2 The 'standard' direction, based on the original direction of Egyptian hieroglyphic and hieratic writing is the right-facing figure: Bonnet, $O L Z 27$ (1924), 554, with many other discussions. Thus in the Hesyre panels the 'private' right-over-left fold is represented in the right-facing figures. On the Khaibausokar false door the right-facing figure similarly has the expected right-over-left fold while the corresponding left-facing figure exhibits the opposite left-over-right fold: cf. for both illustrations: E. L. B. Terrace and Henry G. Fischer, Treasures of Egyptian Art from the Cairo Museum (London, 1970), 35, 39. The subsequent development of these conventions is complex and beyond the scope of this communication.

3 Muzeum Narodowe MNK-XI-999.

4 Vollständige Neuübersetzungen: W. Schenkel, Memphis, Herakleopolis, Theben (Wiesbaden, I 965), 62-4, Nr. 42; M. Lichtheim, Ancient Egyptian Literature I (Berkeley, 1973), 87-8. Teilkommentare: H. G. Fischer, Kush ro (1962), 333-4 [zu Z.5-6; 10; 12]; Schenkel, ZÄS 92 (1965), 63 [zu Z.5; 1 2]; A. Roccati, RSO 42 (1967), 70-1 [zu Z.8-13]; M. Gilula, RdE 20 (1968), 55-6 n. 6 [zu Z.4]; Schenkel, Die Bewässerungsrevolution im Alten Ägypten (Mainz, 1978), 36 [zu Z.9-13]; H. G. Fischer, in Studies in Ancient Egypt, the Aegean, and the Sudan. Studies in honor of Dows Dunham eds. W. K. Simpson and W. M. Davis, (Boston, r 98r), 66(e) [zu Z.ro]; D. Franke, Altägyptische Verwandtschaftsbezeichnungen im Mittleren Reich (Hamburg, 1983), 301 [ [zu Z.5]; 215 [zu Z. IO-II].

5 Op. cit. 6.

7 Bei Gilula, RdE 20 (1968), 55-6 n. 6. 
Die ersten beiden Vorschläge sind zweifellos inhaltlich unbefriedigend, aber auch Grolls Übersetzung kann nicht überzeugen; besonders die Wiedergabe des zweiten Teils als $n r h$ nnk $n$ hsm $n \cdot j$ rmn 'because of my knowledge of one who bent (his) arm to me' ist sehr gezwungen und entspricht kaum der Phraseologie dieser Texte. Überdies ist die angenommene Konstruktion mit $n n k$ hier äußerst zweifelhaft: Man sollte in Parallele zu $n$ $\underline{d} d(\cdot j)$ doch $n r h(\cdot j)$ erwarten, zumal der Possessivausdruck der ersten Person in keiner Weise hervorgehoben ist. Auch andere Belege für $n n k$ nach Infinitiv ${ }^{1}$ sind nicht völlig zweifelsfrei: Der von Gardiner (Egyptian Grammar ${ }^{3}$, 225, 309) zitierte Fall könnte auch $n$ $r w \underline{d} n n k(3 \cdot j) h r j b \cdot f$ zu verstehen sein. ${ }^{2}$ Der einzige sichere Beleg bei Gilula (op. cit.) scheint mir C'T III, 224c zu sein: Hier dient $n n k$ (in Variation mit $n \cdot k$-jmjjt) klar der Hervorhebung des Possessivverhältnisses. ${ }^{3}$ Lichtheims Übersetzung hört sich zweifellos am besten an. Sie wäre noch besser, wenn man das etwas gewaltsam wirkende 'and the bending of my arm' mit 'zu, gegenüber einem, der mir den Arm beugte (= mich respektierte)' wiedergäbe. Lichtheim übersetzt allerdings nicht nur-wie Groll- $n$ psg(w) $m$ jrtj passivisch, sondern auch das vorhergehende $n j w s(\cdot j) .{ }^{4} \mathrm{Daß}$ dies einen besseren Sinn macht und es für einen Beamten 'rather pointless' wäre, zu erklären, daß er nicht geraubt habe, stimmt aber nun wirklich nicht. Est ist im Gegenteil für jemanden, der für das Opferfleisch zuständig war, höchst angebracht zu versichern, daß er nichts unterschlagen hatte.

Ich glaube aber, daß auch der erste Teil bei Lichtheim nicht richtig ist. Mein Vorschlag ist, jeweils das $n$ nach $n f r$ und nach $r h$ als Negation zu verstehen. ${ }^{5}$ Im Zusammenhang: '(Ich stahl nicht,) ich spuckte nicht in die Augen eines Guten, ich sprach nicht (belehrend) zu einem Wissenden, ich verletzte nicht einen, der mir den Arm beugte. ${ }^{6}$ Diese Deutung scheint mir vor allem deshalb besser, weil dann eine perfekte Parallelität zwischen $n p s g(\cdot j)$ $m$ jrtj $n n f r$ und $n n k n(\cdot j)$ him $n(\cdot j) r m n$ besteht.

2. Das Wort I ${ }^{\top}$ in $Z .5$ ist von Cerný ${ }^{7}$ als $s m^{3} y(w)$ 'Genossen' verstanden worden. Die Schreibung I erklärt er als von smyt 'Wüste' entlehnt. Schenkel und Lichtheim haben sich der Übersetzung angeschlossen. ${ }^{8}$ Es bietet sich aber eine viel zwanglosere Erklärung an. Zunächst stimmt es nicht, daß der Vogel eher ein 3 als ein tjw sei, wie Černý meint, er unterscheidet sich im Gegenteil deutlich vom Alif-Zeichen (in Z.3; 4; 8; 10; 1 2; 1 3), sowohl in Größe als auch in Kopf- und Rückenform (und ebenso von w). Es kann nur ein tjw-Vogel sein. Auch die Entlehnung des I von zmjt (das laut $W b$. erst im NR mit diesem Zeichen geschrieben wird) bzw. eine Verderbnis aus dem Hieratischen scheinen mir nicht sehr überzeugend. Es wird statt dessen sp; tjw 'Gaubewohner' zu lesen sein, analog etwa zu nwt 'Stadt'-nwtjw 'Städter'. ${ }^{9}$ Die Aufrechtstellung sonst nur liegend geschriebener Zeichen (entgegen ihrer bildlichen Bedeutung) findet sich gerade in der ersten Zwischenzeit und im frühen MR. ${ }^{10}$

3. Die größte Crux der ganzen Inschrift ist der Satz zu Beginn von Z.6. Seit Fischer die

1 S. Gilula, loc. cit.

2 D.h. exakt so, wie Lichtheim die hier in Frage stehende Stelle versteht, s.o.

${ }^{3}$ Für einen noch deutlicheren Fall im späten Mittelägyptisch s. meine Ägyptischen Biographien der 22. und 23. Dynastie (Wiesbaden, I 985 ) $, 464, Z .7-8$ und 66: Hier besteht ein klarer Gegensatz ‘... ich meinerseits ( $j n n k$ ) .... schon mein Vater ....'!

${ }_{4}$ Zum Verb vgl. J. Osing, Die Nominalbildung des Ägyptischen (Mainz, I 976), II, 765 (929). Ein weiterer Beleg in CT VI, I (Sp. 472).

5 S. dazu die Belegsammlung bei B. Gunn, Studies in Egyptian Syntax (Paris, 1924), 88.

${ }^{6}$ Als Zeichen der Ehrfurcht, s. Wb. III, 23 I, 3.

7 Op. cit. 6 (d).

8 Schenkel, Memphis, Herakleopolis, Theben, 63 (d) möchte I allerdings eher als aus hieratischem ${ }^{\mathrm{I}} \mathrm{verderbt}$ erklären.

${ }^{9} \mathrm{Vgl}$. auch $W b$. Iv, 99, I2 (dort von Göttern).

10 Belege bei J. Polotsky, Zu den Inschriften der II. Dynastie (Leipzig, 1929) §33 und Schenkel, Frühmittelägyptische Studien (Bonn, 1962), §3. 

Lesung von i als $\check{s}$ zot (statt $j t j \cdot j$ wie bei Černý) geklärt hat, ${ }^{1}$ ist die Stelle dreimal übersetzt
worden:

Fischer: 'My forehead and feather nodded assent.'

Schenkel: 'Weiter habe ich meine Feder nach vorn geneigt(?).'

Lichtheim: ${ }^{5}$ 'I have bowed brow and feather.'

Diese Übersetzungen basieren alle auf Fischers (zurückhaltendem) Vorschlag, daß hier an die Feder als Zeichen der Friedfertigkeit gedacht sein könnte. Er verweist dafür auf eine Bemerkung von Clère, ${ }^{6}$ der gezeigt hat, daß die (Straußen) Feder (in den Händen besiegter Feinde) ein Zeichen der Unterwerfung sein kann ebenso wie (in den Händen von Boten) ein Zeichen friedlicher Absichten. ${ }^{7}$ Auf unsere Stelle übertragen hieße das, $M r r$ habe in seinem sozialen Verhalten dauernd 'die weiße Fahne gezeigt', also Friedfertigkeit, wenn nicht gar Unterwerfung signalisiert. Dieser Gedanke widerspricht allem, was wir von der Phraseologie ägyptischer Biographien und ägyptischer Ethik überhaupt wissen. Friedfertigkeit und soziales Wohlverhalten wird dort immer nur aus einer Position der Stärke heraus (lobend) beschrieben. Ganz abgesehen davon läßt sich das angebliche hist 'Stirn' (Fischer und Lichtheim) bzw. 'Vorderseite' (Schenkel) mit šwt kaum in sinnvoller Weise verbinden. ${ }^{8}$ Man wird daher nach einer anderen Lösung suchen müssen.

Mein Vorschlag geht davon aus, daß hier für histj oder genauer $h i t j(\cdot j)$ 'mein Herz' steht, eine determinativlose Schreibung, die ich weiter leider nicht belegen kann. ${ }^{9}$ Im Zusammenhang hieße die Stelle dann: 'Mein Herz neigte (= ließ sinken) die Feder.' Das kann sich nur auf die Szenerie des Totengerichts beziehen, wo das Herz gegen die Maatfeder abgewogen wird. ${ }^{10}$ Weiter läßt sich daraus schließen, daß bei diesem Wiegevorgang nicht die guten Taten gewogen werden (und 'schwer' wiegen sollen), sondern im Gegenteil die Sünden, das Herz mithin im Idealfall leicht, 'unbelastet' ist. ${ }^{11}$

Dies ist in der Tat auch das Bild, daß die wenigen Texte bieten, die sich etwas deutlicher zu diesem Punkt äußern, wie die Untersuchung durch Clère ergeben hat. ${ }^{12}$ Sein Ergebnis ist durch Spiegel ${ }^{13}$ dahingehend relativiert worden, daß der Wiegevorgang beim Totengericht aus mehreren Akten bestand, wobei sowohl die Sünden als auch die guten Taten gewogen wurden. ${ }^{14}$ Dem hat sich-wohl zu Recht - auch Seeber angeschlossen, ${ }^{15}$ obwohl zumindest in älterer Zeit das Abwiegen der guten Taten nicht durch textliche Aussagen nachgewiesen werden kann. Die erste Vorstellung-das Abwiegen der Sünden-wird in jedem Fall dominierend gewesen sein: dafür spricht ja auch die 'negative Konfession' und eben die Tatsache, daß das Herz in den Darstellungen (wie in dem hier besprochenen Text) gegen die Maatfeder abgewogen wird. Es wäre doch sehr verwunderlich, wenn eine Feder nicht zugleich auch als Zeichen der Leichtigkeit verstanden worden wäre.

1 Kush 10 (1962), 334 .

2 Loc. cit.

${ }^{3}$ Memphis, Herakleopolis, Theben, 63.

4 In seiner Anmerkung e: 'Wörtl.: Ich habe die Vorderseite meiner Feder geneigt; d.h. ich war friedlich?'

5 Op. cit. 87. ${ }^{6} M D A I K$ I 6 (1958), 4I-2.

7 Ganz entsprechend der 'weißen Fahne' als Zeichen der Kapitulation oder des Parlamentärsstatus.

8 Die Feder als Zeichen der Friedfertigkeit muß ja gerade in der Hand getragen werden; an der Stirn ist sie das Abzeichen des Kriegers.

9 Immerhin könnte man auf die allgemein knappere Determinierung der Inschriften der ersten Zwischenzeit verweisen, vgl. Schenkel, Frühmittelägyptische Studien, § го.

${ }_{10}$ Zum Verb hnn im Sinne von 'die Waagschale neigen, sinken lassen' vgl. $W b$. II, 494, I $3=$ pHarris I, $26, \mathrm{I} 2$ (dort allerdings ohne Objekt gebraucht).

11 Aus den (späteren) Darstellungen (mit Herz und Feder bzw. Maat im Gleichgewicht) geht ja nicht hervor, ob Sünden oder gute Taten (oder beides) gewogen werden.

12 BIFAO 30 ( I93I), $430 \mathrm{ff}$.

13 Die Idee vom Totengericht in der ägyptischen Religion (Glückstadt, 1935), $68 \mathrm{ff}$.

14 Wobei dann-bei gutem Ausgang-eine Umkehrung der Gewichtsverhältnisse erforderlich ist.

15 Untersuchungen zur Darstellung des Totengerichts im Alten Ägypten (München, 1976), 80 ff. 
Die Richtigkeit dieser Deutung vorausgesetzt, wäre diese Stelle der älteste Beleg für den Wiegevorgang im Totengericht, ${ }^{1}$ und für ihre Richtigkeit spricht schließlich auch der Zusammenhang mit dem Vorhergehenden: Der Satz folgt auf $j w z b j \cdot n(\cdot j) r j m i h$ 'ich erreichte den jmih-Status'.

Dennoch möchte ich diesen Vorschlag nur mit einiger Zurückhaltung vorbringen: Die Schreibung des angeblichen $h \operatorname{ti} j(\cdot j)$ ohne Herz-Determinativ ist-wie erwähntebensowenig belegt wie die Bezeichnung des Gegengewichts auf der Waage schlicht als šwt 'die Feder' statt durch ' $m$ s $t$ '.

4. Nachdem $M r r$ in Z.I-7 zunächst von seiner Tätigkeit als Schlachtervorsteher und dann von seinem Charakter und seinem tadellosen sozialen Verhalten gesprochen hat, beginnt in Z.7 mit jw grt der zweite Abschnitt der Biographie, der (sehr viel konkreter) seine Taten für Stadt und Mitbürger schildert. Der Beginn dieses Abschnittes ist folgendermaßen übersetzt worden:

Černý (op. cit.): 'I also feared to become a kt swt of this town.'

Schenkel (op. cit.): 'Weiter aber war Furcht entstanden in einer anderen (Stadt), mich aber rühmte diese Stadt.'

Lichtheim (op. cit.): 'And when fear had arisen in another town, this town was praised.'

Auf Černýs Deutung (von ihm selbst nur sehr zweifelnd vorgebracht) braucht man wohl nicht weiter einzugehen. Schenkel und Lichtheim betrachten beide $\{$ f als das nur einmal belegte angebliche stw 'bewundern, preisen', das vom $W b{ }^{3}$ wohl richtig zu stwt 'rühmen' gestellt, vorsichtshalber aber als eigenes Wort angeführt wird. Die Hauptschwäche ihrer Übersetzung (abgesehen von syntaktischen Problemen in Lichtheims Version) sehe ich darin, daß das (bezugslose) kjtj im ersten Teilsatz steht, worauf dann erst $n w t$ tn folgen soll, also genau umgekehrt der zu erwartenden, logischen Anordnung. Außerdem ist der Sinn dieser Übersetzungen alles andere als befriedigend; der Gegensatz 'Furcht' und 'rühmen, preisen' ist nicht sehr naheliegend.

Auch hier läßt sich eine völlig andere Deutung geben: Ich lese die Stelle $j w$ grt sndw $w(\cdot j)$ $h p r(w)(r) m k t$ swtj nwt $(\cdot j)$ tn 'Furcht vor mir entstand (nur) um zu schützen und mächtig zu machen diese (meine) Stadt'. In dem von Schenkel und Lichtheim als stw verstandenen Verb sehe ich den Infinitiv von swtj 'groß, mächtig machen' ${ }^{4}$ Die Gruppe als ganze $h \operatorname{pr}(w)$ zu lesen: ${ }^{5}$ dann wäre ein durch Haplographie ausgefallenes $r$ zu ergänzen. In dieser Zeit ist allerdings auch die Schreibung 蝞 für hpr belegt; ${ }^{6}$ so wäre nicht einmal diese kleine Ergänzung nötig.

KarL JANSEN-WinkELN 